\title{
An X-Band low-power and low-phase-noise VCO using bondwire inductor
}

\author{
K. Hu, F. Herzel, and J. C. Scheytt \\ IHP GmbH, Im Technologiepark 25, 15236 Frankfurt (Oder), Germany
}

\begin{abstract}
In this paper a low-power low-phase-noise voltage-controlled-oscillator (VCO) has been designed and, fabricated in $0.25 \mu \mathrm{m} \mathrm{SiGe} \mathrm{BiCMOS}$ process. The resonator of the VCO is implemented with on-chip MIM capacitors and a single aluminum bondwire. A tail current filter is realized to suppress flicker noise up-conversion. The measured phase noise is $-126.6 \mathrm{dBc} / \mathrm{Hz}$ at $1 \mathrm{MHz}$ offset from a $7.8 \mathrm{GHz}$ carrier. The figure of merit (FOM) of the VCO is $-192.5 \mathrm{dBc} / \mathrm{Hz}$ and the $\mathrm{VCO}$ core consumes $4 \mathrm{~mA}$ from a $3.3 \mathrm{~V}$ power supply. To the best of our knowledge, this is the best FOM and the lowest phase noise for bondwire VCOs in the X-band. This VCO will be used for satellite communications.
\end{abstract}

\section{Introduction}

For satellite communications, such as HDTV, internet-viasatellite and digital video broadcasting service (DVB-RCS), a low-phase-noise VCO is a prime requirement for the frequency synthesizer. Following the specifications given in (Follmann et al., 2008), phase noise must be lower than $-110 \mathrm{dBc} / \mathrm{Hz}$ at $1 \mathrm{MHz}$ offset from the carrier frequency. With the integrated resonator, this specification is hard to meet, particularly if the performance must be guaranteed over a wide tuning range. A VCO phase noise of $-120 \mathrm{dBc} / \mathrm{Hz}$ at $1 \mathrm{MHz}$ offset is, therefore, highly desirable.

With respect to cost, silicon compares favourably to III-V technologies. However, it is difficult to reach the phase noise specification with a fully integrated VCO for silicon technology due to the poor quality factor of the on-chip inductor. Many studies have been presented on minimizing VCO phase noise, e.g. Hegazi (2001); Ferndahl (2005). However, the overall performance of these VCOs, e.g. power con-

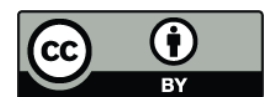

Correspondence to: $\mathrm{K} . \mathrm{Hu}$ (hu@ihp-microelectronics.com) sumption, tuning range, phase noise performance, can still not satisfy the specifications. In order to reduce phase noise and maintain relatively low power consumption, bondwire inductors have been used (Craninckx et al., 1995; Kim et al., 2008). By doing so, a figure of merit (FOM) of $-190 \mathrm{dBc} / \mathrm{Hz}$ has been reported so far.

A differential CMOS VCO implemented with nMOSFETs and pMOSFETs was presented in (Craninckx et al., 1997). Unlike in VCOs using one transistor type only, in this current re-using topology no mid-point in the inductor is required for biasing purpose. We adopt this topology by using a bondwire inductor instead of an integrated coil. This approach minimizes mismatch in the VCO as only one bondwire is used rather than two in other topologies. Moreover, a tail current filter is introduced to reduce the flicker noise up-conversion from the current source. This approach significantly improves the close-in frequency phase noise of the VCO.

\section{VCO design}

The VCO core employs two nMOSFETs and two pMOSFETs. The combination of NMOS and PMOS transistors gives a negative resistance from both transistor pairs. Consequently, to provide the same negative resistance, the combined NMOS and PMOS structure can efficiently halve the power consumption, which greatly fits the purpose of low power design. Furthermore, by controlling the supply voltage, the signal swing in the VCO core can be well restricted below the transistor breakdown voltage. It ensures a more reliable operation, which is a very important requirement for satellite communications (Tiebout, 2006).

Another convenient feature of this VCO topology is that only one inductor is required for the resonator. This eliminates the inductance mismatch which appears in topologies using two bondwire inductors. The mismatch of resonator inductors in these topologies will result in asymmetric signal

Published by Copernicus Publications on behalf of the URSI Landesausschuss in der Bundesrepublik Deutschland e.V. 


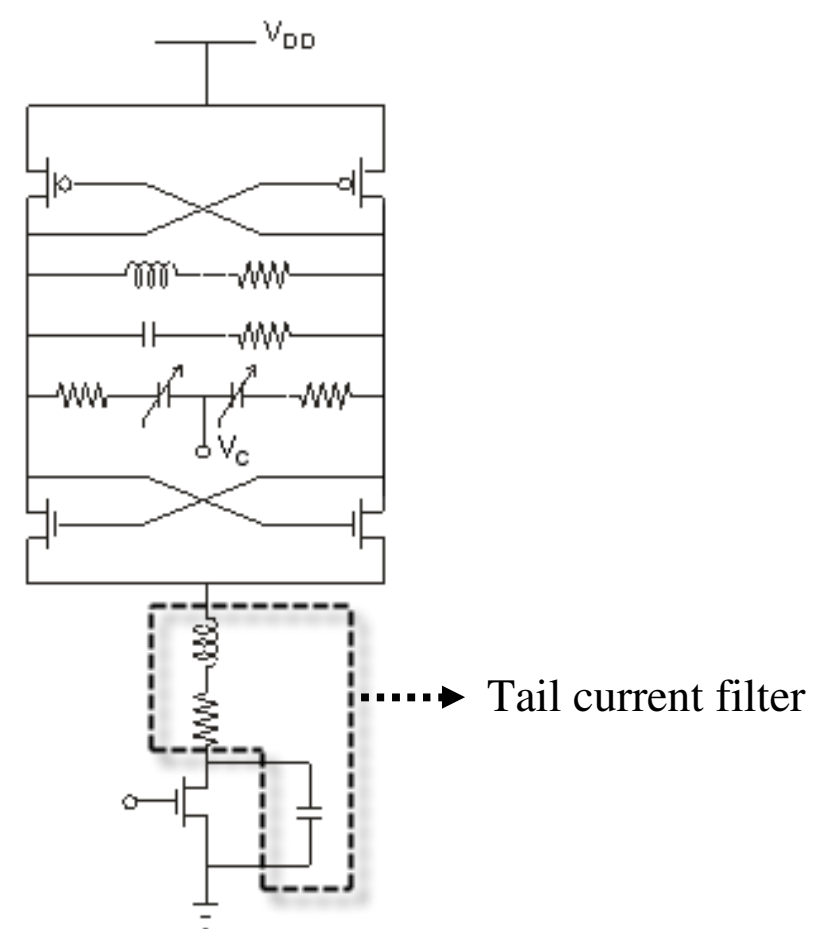

Fig. 1. Schematic of the X-Band VCO.

swing across the tank and, consequently, degenerate the oscillator phase noise.

Additionally, the phase noise close to the carrier frequency is mainly dominated by the flicker noise up-conversion, i.e. flicker noise from current source and VCO core transistors. As a result of the odd mode virtual ground at the differential common mode node, the flicker noise from current source is up-converted to $2 \cdot f_{0}$ and mixed down to $f_{0}$ (oscillation frequency), because of the differential pairs mixing action. The up-converted flicker noise will be delivered to the resonator as AM noise (Rael et al., 2000), and the AM noise will be converted to FM noise in the varactor (Muer et al., 2000). Thus, for the suppression of flicker noise from current source, tail current filtering technique (Hegazi et al., 2001) is introduced to short the 2 nd harmonic at common mode node. As a result, the close-in frequency phase noise can be largely improved.

\section{Bondwire modelling}

As mentioned above, there are many studies about improving the phase noise performance for VCO designs, but most of them cannot meet both the low phase noise and the low power consumption requirements. The modified Leeson's Formula (Masini et al., 2001):

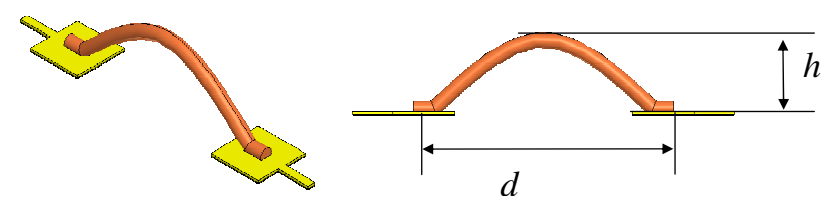

Fig. 2. Bondwire model. $h$ is wire loop height, $d$ is pad distance.

Table 1. EM simulation results for bondwire.

\begin{tabular}{ll}
\hline Pad Distance & Wire Radius $20 \mu \mathrm{m}$ \\
\hline $250 \mu \mathrm{m}$ & $325 \mathrm{pH}$ \\
$300 \mu \mathrm{m}$ & $386 \mathrm{pH}$ \\
$400 \mu \mathrm{m}$ & $502 \mathrm{pH}$ \\
$500 \mu \mathrm{m}$ & $635 \mathrm{pH}$ \\
$600 \mu \mathrm{m}$ & $751 \mathrm{pH}$ \\
\hline
\end{tabular}

$L(\Delta \omega)=10 \cdot \log \left\{\frac{2 \cdot F \cdot k \cdot T}{P_{s}} \cdot\left[1+\left(\frac{1}{2 Q} \cdot \frac{\omega_{c}}{\Delta \omega}\right)^{2}\right]\right.$

$\left.\cdot\left(1+\frac{\Delta \omega_{1 / f^{3}}}{|\Delta \omega|}\right)+\frac{\pi^{2}}{2} \cdot\left(\frac{K_{v} \cdot V_{m}}{\Delta \omega}\right)\right\}$,

gives a guideline for VCO optimization. Here $F$ is the noise figure of the transistor, $\Delta \omega$ is the offset frequency, $\omega_{c}$ is the center frequency, $P_{S}$ is the power across the tank, $Q$ is the loaded $Q$ of the tank, $K_{v}$ is the VCO gain and $V_{m}$ is the total low frequency noise e.g. noises from DC sources or tuning lines. It is evident from Eq. (1) that the most efficient way of reducing VCO phase noise is to increase the loaded $Q$ of the resonator tank. For the $\mathrm{LC}$ resonator $\mathrm{VCO}$, the loaded $Q$ of the tank can be written as:

$Q=\frac{Q_{L} \cdot Q_{C}}{Q_{L}+Q_{C}}$.

In Eq. (2), $Q_{L}$ is the quality factor of the tank inductor, and $Q_{C}$ is the quality factor of the tank capacitor. As a result of using the MIM capacitors, $Q_{C}$ is generally much higher than $Q_{L}$. Thus, the loaded $Q$ of the tank is mainly determined by the inductor quality factor. However, due to the lossy silicon substrate, on-chip inductor in the X-Band can only have a quality factor around 10. A different choice for on-chip inductors takes advantage of the parasitic inductance, which is usually associated with bondwire in IC packaging. The series resistance of the bondwire is very low, which leads to a high quality factor. Furthermore, the parasitic capacitance value is given by the bond pads. Thus, by using optimized bondpad size the bondwire inductor can have a very high self-resonance frequency. Figure 2 shows the structure of the bondwire inductor. The radius of the bondwire is $12.5 \mu \mathrm{m}$. The pad distance and wire loop height are defined as design parameters to simulate bondwire inductance and quality factor. In order to obtain accurate prediction results, full wave 


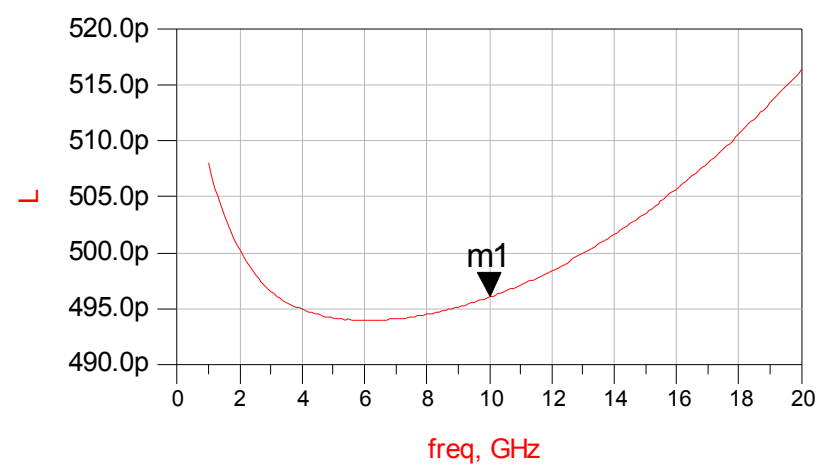

Fig. 3. Inductance for $480 \mu \mathrm{m}$ length bondwire (EM simulation).

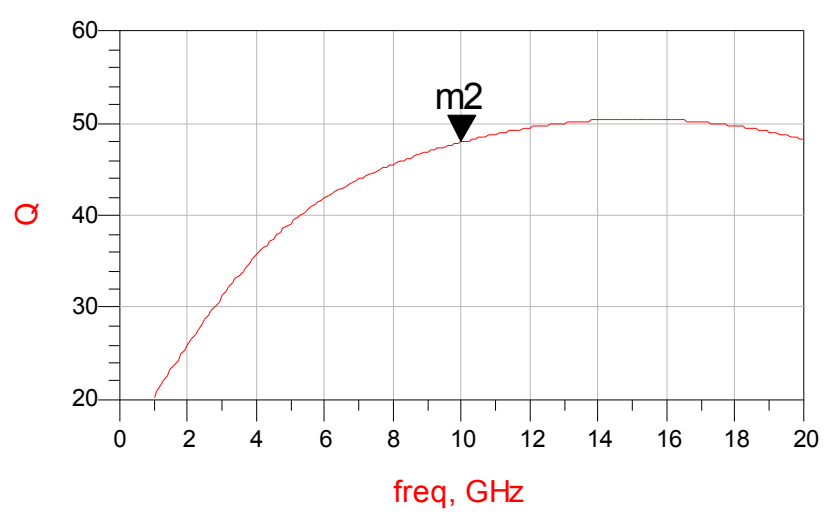

Fig. 4. Quality factor for $480 \mu \mathrm{m}$ length bondwire (EM simulation).

EM simulator (Ansoft HFSS v11) is used for the bondwire simulations.

Table 1 shows the simulated inductance for different bondwire lengths. The size of the bondpad affects the inductor self-resonance frequency significantly, which is caused by the parasitic capacitance between pad and substrate.

For the requirement of the X-band VCO design, a $500 \mathrm{pH}$ inductor corresponds to an aluminum bondwire with the length of $480 \mu \mathrm{m}$. With optimized bondpad size, the simulated quality factor can reach 48 at $10 \mathrm{GHz}$. Figures. 3 and 4 show the simulated inductance and quality factor for the bondwire inductor used in the X-band VCO design.

\section{Simulation results}

As shown in Fig. 5, the simulated result demonstrates $8 \mathrm{~dB}$ phase noise improvement at $100 \mathrm{kHz}$ offset and $4 \mathrm{~dB}$ phase noise improvement at $1 \mathrm{MHz}$ offset with the tail current filtering technique. The simulated phase noise for the final VCO design is $-128.3 \mathrm{dBc} / \mathrm{Hz}$ at $1 \mathrm{MHz}$ offset and $-105 \mathrm{dBc} / \mathrm{Hz}$ at $100 \mathrm{kHz}$ offset from a $10.2 \mathrm{GHz}$ carrier. Obviously, the up-conversion of flicker noise has been greatly reduced by introducing the tail current filtering structure.

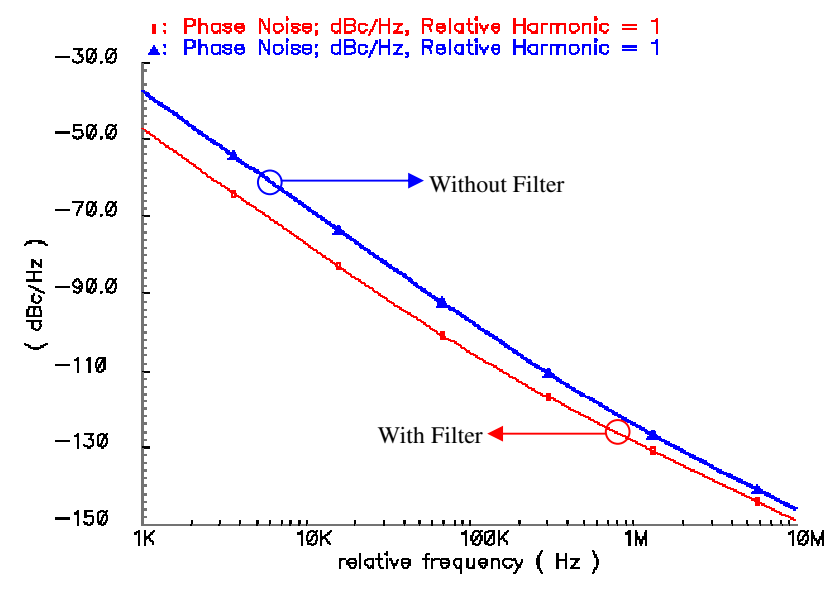

Fig. 5. Phase noise with and without tail current filter.

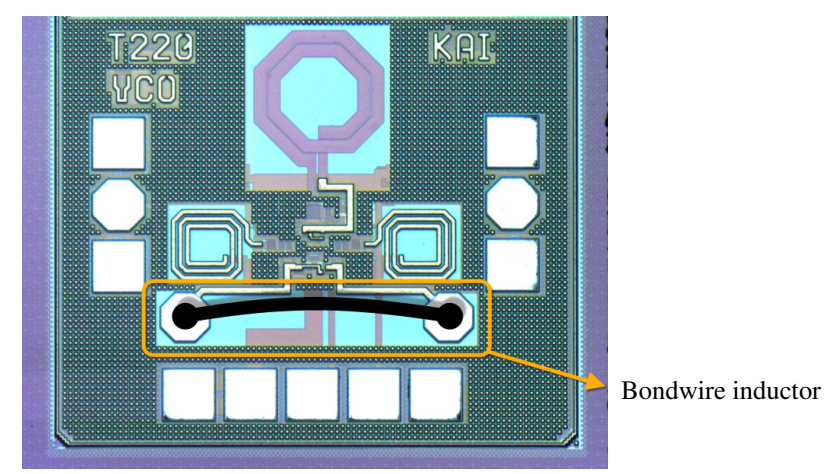

Fig. 6. Chip photograph.

\section{Measurement results}

A photograph of the VCO chip is shown in Fig. 6. The VCO operates from a $3.3 \mathrm{~V}$ supply and consumes $4 \mathrm{~mA}$ current. With the $50 \mathrm{Ohm}$ output buffer, the VCO delivers an output power from -1 to $2 \mathrm{dBm}$ over the whole tuning range. The measured tuning frequency and output power is shown in Fig. 7.

The VCO phase noise is measured by Aeroflex phase noise meter with the delay line method. As shown in Fig. 8, the phase noise at $1 \mathrm{MHz}$ offset is $-126.6 \mathrm{dBc} / \mathrm{Hz}$ and shows a very good matching with the simulation results. At $100 \mathrm{kHz}$ offset from the $7.8 \mathrm{GHz}$ carrier, the measured phase noise is $-100 \mathrm{dBc} / \mathrm{Hz}$. This relative high phase noise at $100 \mathrm{kHz}$ is due to the flicker noise of the MOSFETs.

The figure of merit for a VCO is defined by Eq. 3 (Tiebout, 2006), where $\omega_{0}$ is the angular oscillation frequency, $L(\Delta \omega)$ is the phase noise at offset $\Delta \omega$ and $P_{\text {diss }}$ is the dc power consumption $(\mathrm{mW})$ of the VCO. The measured X-band VCO has a FOM of $-192.5 \mathrm{dBc} / \mathrm{Hz}$. To the best of the authors' knowledge, this is the best FOM among published X-band VCOs based on silicon technology. 
Table 2. Performance of reported VCO.

\begin{tabular}{llllllll}
\hline & $\begin{array}{l}F_{C} \\
(\mathrm{GHz})\end{array}$ & $\begin{array}{l}P_{\text {out }} \\
(\mathrm{dBm})\end{array}$ & $\begin{array}{l}\mathrm{L} @ 1 \mathrm{MHz} \\
(\mathrm{dBc} / \mathrm{Hz})\end{array}$ & $\begin{array}{l}\text { Offset } \\
(\mathrm{MHz})\end{array}$ & $\begin{array}{l}\text { FOM } \\
(\mathrm{dB})\end{array}$ & Technology & Topology \\
\hline H. Jacobsson/RFICS & 11.8 & -7 & -123 & 1 & -183.9 & $0.5 \mu \mathrm{m} \mathrm{SiGe}$ & 4× coulpled VCO array \\
H. Jacobsson/RFICS & 5.9 & -9 & -126 & 1 & -185.5 & $0.25 \mu \mathrm{m} \mathrm{SiGe}$ & Coulpled Colpitts \\
Orsatti/CICC 1999 & 0.9 & $\mathrm{NA}$ & -112.7 & 0.1 & -185.3 & $\mathrm{NA}$ & External LC resonator \\
Svelto/CICC 2000 & 1.9 & $\mathrm{Na}$ & -148 & 3 & -187 & $\mathrm{NA}$ & Bondwire VCO \\
K. J. Kim/ISED 2008 & 1.7 & $\mathrm{NA}$ & -135.3 & 1 & -190 & $0.18 \mu \mathrm{m}$ CMOS & Bondwire VCO \\
This work & 8.8 & 1.92 & -125.3 & 1 & -192.5 & $0.25 \mu \mathrm{m} \mathrm{SiGe}$ & CC/Bondwire \\
\hline
\end{tabular}

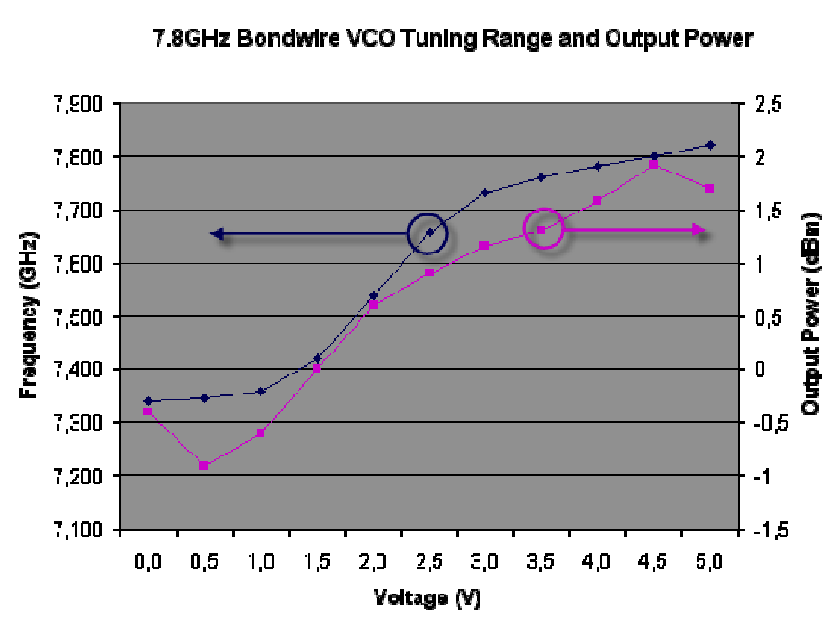

Fig. 7. Measured output power and tuning curve.

$\mathrm{FOM}=L(\Delta \omega)-20 \cdot \log \left(\frac{\omega_{0}}{\Delta \omega}\right)+10 \cdot \log \left(\frac{P_{\mathrm{diss}}}{1 \mathrm{~mW}}\right)$

Table 2 lists the performance of state-of-art VCOs based on silicon technology.

\section{Conclusions}

We have presented an X-band bondwire VCO in $0.25 \mu \mathrm{m}$ SiGe BiCMOS technology. The bondwire was optimized by using full-wave EM simulation. The tail current noise is compensated by an integrated low pass filter. Due to the use of the complementary MOSFETs, only one bondwire is required. This improves the symmetry of the differential circuit. The presented VCO achieved a phase noise of less than $-125 \mathrm{dBc} / \mathrm{Hz}$ at $1 \mathrm{MHz}$ offset. The tuning range is from 7.4 to $7.8 \mathrm{GHz}$. The figure of merit is $-192.5 \mathrm{dBc} / \mathrm{Hz}$. To the authors' knowledge, this is the best value reported so far for silicon-based X-band VCOs. In a future design, the complementary MOSFETs will be replaced with NPN and PNP hetero-bipolar transistors (HBTs) in a complementary SiGe BiCMOS technology (Heinemann et al., 2003). This will reduce flicker noise (Niu, 2005) and improve radiation hardness (Cressler et al., 1998).

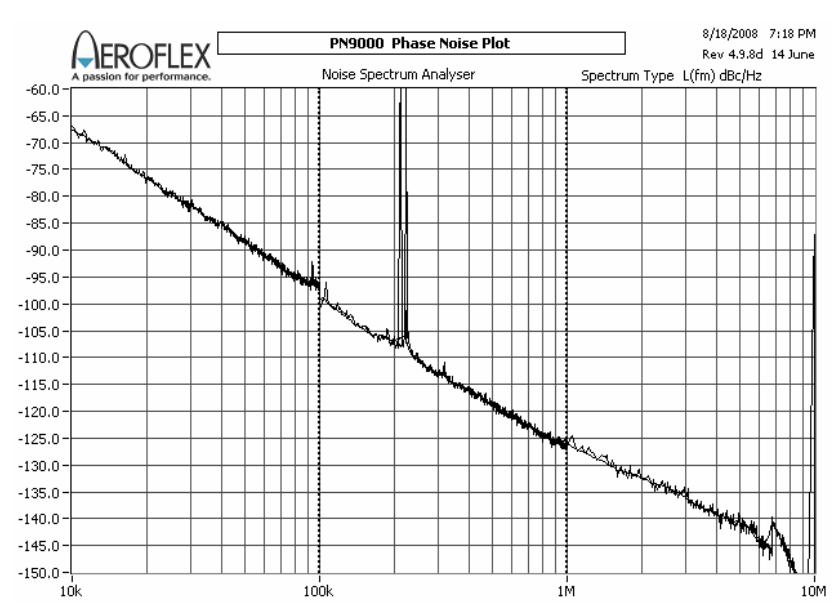

Fig. 8. Measured phase noise for $7.8 \mathrm{GHz}$ VCO.

Acknowledgements. This work was supported by the European Space Agency (ESA) and the German DLR (Deutsches Zentrum für Luft- und Raumfahrt). The authors thank the IHP technology team for the fabrication of the test chip.

\section{References}

Follmann, R., Köther, D., Kohl, T., Engels, M., Podrebersek, T., Heyer, V., Schmalz, K., Herzel, F., Winkler, W., Osmany, S., and Jagdhold, U.: A single SiGe chip fractional-N 275 MHz. . $20 \mathrm{GHz}$ PLL with integrated $20 \mathrm{GHz}$ VCO, IEEE MTTS International Microwave Symposium, Atlanta, 355-358, June, 2008.

Hegazi, E., Sjoland, H., and Abidi, A.: A Filtering Technique to Lower Oscillator Phase Noise, IEEE International Solid-State Circuits Conference, 364-365, 2001.

Jacobsson, H., Hansson, B., Berg, H., and Gevorgian, S.: Very Low Phase-Noise Fully-Integrated Coupled VCOs, Radio Frequency Integrated Circuits Symposium, 467-470, 2002.

Bao, M., Li, Y., and Jacobsson, H.: A $21.5 / 43$ GHz Dual-Frequency Balanced Colpitts VCO in SiGe Technology, IEEE Journal of Solid-State Circuits, 1352-1355, 2004.

Zirath, H., Jacobsson, H., Bao, M., Ferndahl, M., and Kozhuharov, R.: MMIC-Oscillator Designs for Ultra Low Phase Noise, IEEE 
Compound Semiconductor Integrated Circuit Symposium, 204207, 2005.

Ferndahl, M. and Zirath, H.: Broadband $7 \mathrm{GHz}$ VCO in mHEMT Technology, in: Proc. Asia-Pacific Microwave Conference, 4, December, 2005.

Craninckx, J. and Steyaert, M.: A 1.8-GHz CMOS low-phasenoise voltage-controlled oscillator with prescaler, IEEE Journal of Solid-State Circuits, 1474-1482, December, 1995.

Kim, K., Ahn, K. H., and Lim, T. H.: Low Phase Noise Bond Wire VCO for DVB-H, 4th IEEE International Symposium on Electronic Design, Test and Applications, 103-106, 2008.

Craninckx, J., Steyaert, M., and Miyakawa, H.: A Fully Integrated Spiral-LC CMOS VCO Set with Prescaler for GSM and DCS1800 Systems, in: Proc. IEEE Custom Integrated Circuits Conference, 403-406, 1997.

Tiebout, M.: Low Power VCO Design in CMOS, Springer Berlin Heidelberg, 11 and 74, 2006.

Rael, J. J. and Abidi, A. A.: Physical Process of Phase Noise in Differential LC Oscillators, in: Proc. Custom Integrated Circuit Conference, 569-572, May, 2000.
Muer, B. D., Borremans, M., Steyaert, M., and Puma, G. L.: A 2-GHz Low-Phase Noise Integrated LC-VCO Set With FlickerNoise Upconversion Mechanism, IEEE j. Solid-State Circuits, 1034-1038, July, 2000.

Hegazi, E., Sjoland, H., and Abidi, A: A Filtering Technique to Lower Qscillator Phase Noise, ISSCC, Session 24, 23.4, 2001.

Masini, L., Pozzoni, M., Caliumi, A., Tomasini, L., Morigi, D., and Lemaire, F.: A Fully Integrated Silicon-Germanium XBand VCO, online available: http://amsacta.cib.unibo.it/archive/ 00000048/01/G_13_2.pdf, 2001.

Heinemann, B., Barth, R., Bolze, D., Drews, J., and Formanek, P.: A complementary BiCMOS technology with high speed npn and pnp SiGe:C HBTs, IEEE International Electron Devices Meeting, 5.2.1-5.2.4, 2003.

Niu, G.: Noise in SiGe HBT RF Technology: Physics, Modeling, and Circuit Implementations, in: Proc. IEEE, 93, 1583-1597, 2005.

Cressler, J. D.: SiGe HBT technology: a New Contender for Si based RF and Microwave Circuit Applications, IEEE Transactions on Microwave Theory and Techniques, 46, 572-589, 1998. 